\title{
GIS-Based Risk Mapping of Cutaneous Leishmaniasis: A Survey in an Endemic Area of Central Iran
}

\section{Mohammad Maracy ( mrmaracy@yahoo.co.uk)}

Skin Diseases and Leishmaniasis Research Center, Department of Epidemiology \&amp; Biostatistics, School of Health, Isfahan University of Medical Sciences, Isfahan, Iran. I

\section{Fariba Jaffary}

Skin Diseases and Leishmaniasis Research Center, Isfahan University of Medical Sciences, Isfahan, Iran. Skin and Stem Cell Research Center, Tehran University of Medical Sciences, Tehran, Iran.

\section{Afshin Ebrahimi}

Environment Research Center, Research Institute For Primordial Prevention Of Noncommunicable Disease, Isfahan University Of Medical Sciences, Isfahan, Iran

\section{Fatemeh Sokhanvari}

Skin Diseases And Leishmaniasis Research Center, Isfahan University Of Medical Sciences, Isfahan, Iran

\section{Asieh Heidari}

Skin Diseases And Leishmaniasis Research Center, Isfahan University Of Medical Sciences, Isfahan, Iran

\section{Hanieh Sharifian Koupaiee}

Skin Diseases And Leishmaniasis Research Center, Isfahan University Of Medical Sciences, Isfahan, Iran

\section{Reza Fadaei}

Isfahan Provincial Health Center, Department Of Disease Control, Isfahan University Of Medical Sciences, Isfahan, Iran

\section{Javad Ramazanpour}

Isfahan Provincial Health Center, Department Of Disease Control, Isfahan University Of Medical Sciences, Isfahan, Iran

\section{Malihe Moazeni}

Student Research Committee And Department Of Environmental Health Engineering, School Of Health, Isfahan University Of Medical Sciences, Isfahan, Iran

\section{Research Article}

Keywords: Cutaneous Leishmaniasis, Geographical Information System, Hot spot analysis

Posted Date: March 31st, 2021

DOI: https://doi.org/10.21203/rs.3.rs-306531/v1

License: (c) (i) This work is licensed under a Creative Commons Attribution 4.0 International License. Read Full License 


\section{Abstract}

Introduction: [Cutaneous Leishmaniasis (CL)] is Vector-borne infectious disease that is affected by various environmental agents. The main objective of this study was to determine the spatial distribution of $\mathrm{CL}$ incidence by using [Geographical Information System (GIS)].

Materials and Methods: This is a cross-sectional study that was conducted during 5 years from 2014 to 2018 in Isfahan, Iran. We used the required data on each leishmaniasis patient that were recorded from 44 counties of Isfahan in databases and archive of provincial Health Centre. We used GIS for determining the incidence of CL in the high-risk foci. Moran index was used to identify high risk points (clustering in similar values) compared to the values of neighborhood points. Hot spot analysis was conducted by Getis-Ord-Gi.

Results: The highest incidence of the disease occurred in the age group of 18-64 years and 61.6\% of patients were male. According to seasonal distribution, autumn (58.6\%) had the highest frequency. Time-trend of incidence showed that it had both decreasing and increasing, and there was a sudden upward trend of disease in 2018 except only two counties. The hot spots were involved the central areas of the Isfahan province slightly toward to the north and southeast of the province. Moran index showed that the differences for all points were not significant (p-value>0.05).

Conclusions: Varzaneh (placed in southeast of Isfahan) was the hottest spot and had the worst position for leishmaniasis compared to all years and all cities.

\section{Introduction}

Leishmaniasis is Vector-borne infectious disease that is affected by various environmental agents, such as distribution of reservoir hosts and vectors of leishmaniasis. Among diseases spread by insects, leishmaniasis is endemic in 88 countries on four continents ( 16 developed countries and 72 developing countries) (Anker, et al 2000).

The disease can be presented in three basic clinical forms: cutaneous, mucocutaneous or visceral leishmaniasis. A common estimate of the worldwide incidence reported 1 to 2 million clinical new cases a year and the risk of disease is about 350 million and have 20,000-40,000 deaths causes each year. Dispite of new diagnosis and treatments of leishmaniasis, but it is now considered as a serious public health problem particularly in developing countries including Iran and has an economic burden on health resources (Cardoso, et al 2019; Mandal, et al 2018; Glans, et al 2018). Leishmaniasis has been reported as one of the most important neglected tropical diseases. It spreaded to new foci due to the influence of many risk factors, especially environmental factors. Evidence shows that the prevalence of this disease in the old and new world is increasing in the early years of this century (Mackey, et al 2014; Masmoudi, et al 2013). Nowadays, development of cost-effective models and [Integrated Management System (IMS)] have important roles to improve the control of cutaneous leishmaniasis, along with epidemiological studies and ecological understanding of disease and the social and environmental risk factors. Also the relevance between environmental variables and epidemiological factors of leishmaniasis, incidence and prevalence, can be useful for next predictions (Golpayegani, et al 2018; Artun 2019). Using maps, spatial analysis and regression techniques can model the statistical relationship between variables in detail. In fact, using this information in epidemiological studies can help public health policy makers in having a better decision for control and prevention of disease (Ostad, et al 2016; Cloots, et al 2020; Blachowski 2016). Leishmaniasis is a disease that accurre in different geographical regions with a focal distribution and [Geographical Information System (GIS)] is one of the 
most important and useful epidemiological tools for determining the geographical areas and populations at risk of the leishmaniasis. In fact it is an applicative software for the spatial distribution of disease quantitatively and qualitatively in forms of mapping for health managing and preventing strategies to control of various diseases, such as [Cutaneous Leishmaniasis (CL)], malaria and HIV infection. A development of the GIS during the two last decades have provided more powerful and efficient tools to investigate spatial patterns to study infectious diseases (Ostad, et al 2016; Shirzadi, et al 2019; Alvar, et al 2012).

The main objective of this study was to determine the spatial distribution of CL incidence in Isfahan province by using GIS software during 2014-2018. We hope to increase awareness and potential guide approaches to address this global health concern through this study.

\section{Materials And Methods}

This is a cross-sectional study that was conducted during 5 years from 2014 to 2018 throughout a leishmaniasis endemic area of Central Iran.

\section{Study Area}

Isfahan Province is one of the provinces of central Iran and consists of 44 counties. It has an area of $115,932 \mathrm{~km}^{2}$ lying within the $31^{\circ} 43^{\prime}$ to $34^{\circ} 22^{\prime} \mathrm{N}$ and $49^{\circ} 38^{\prime}$ to $55^{\circ} 31^{\prime} \mathrm{E}$ with a moderate and dry climateis and is considered as arid and semiarid regions of the Iran. This province has a population of approximately 4,629,312 Inhabitants (Ramezankhani, et al 2017).

\section{Data Collection}

In this study, we used the required data on each leishmaniasis patient, i.e. age, gender, residence (urban, rural), season of disease outbreak, and ulcer zone that were recorded from 44 counties of Isfahan province in the related data bases and also in archive of Provincial Health Centre during a period of 2014-2018. In these past 5years, leishmaniasis patients who were confirmed by a positive skin test and/or parasitological examination, were identified in Isfahan province and subsequantely were eligible for our study.

\section{Statistical And Spatial Analysis}

We used GIS to determine the incidence of CL in the high-risk foci. Geografic latitude and longitude of the area were calculated and digital maps of Isfahan province were designed to show the distribution of CL.

After preparing the project file in Arc-Map software, the maps extracted by ArcGIS version 10.2 (ESRI Inc., Redlands, CA, USA) software. Density maps were designed using Spatial Analyst, GeoStatistical Analyst and appropriate models.

Moran index was used to identify high risk points (clustering in similar values) compared to the values of neighborhood points. Ranging from -1 to +1 . This index is the most useful index for measuring spatial correlation between points, events and phenomena [Kavousi, et al. 2020]. In our study 95\% significance level $(P<0.05)$ was considered. Hot spot analysis was conducted by Getis-OrdGi_which provides a Z score value to indicate whether features with either high or low values cluster spatially (hot and cold spots) (Abedi-Astaneh, et al 2016).

\section{Results}


The incidence of CL during 5 years (2014-2018) in the cities of Isfahan province has shown both upward and downward trend. It should be noted that 14 of 44 cities in Isfahan province have been excluded due to the absence of leishmaniasis patients or having only one patient per 10,000 populations [Table 1]. 
Table 1

Incidence of CL in the cities of Isfahan during 2014-2018(Number of cities is according to statistics and analysis of Isfahan Municipality data).., 1

\begin{tabular}{|c|c|c|c|c|c|c|c|c|c|c|c|}
\hline & \multicolumn{2}{|l|}{2014} & \multicolumn{2}{|c|}{2015} & \multicolumn{2}{|c|}{2016} & \multicolumn{2}{|c|}{2017} & \multicolumn{2}{|c|}{2018} \\
\hline & & $\mathrm{n}^{\mathrm{y}, 2}$ & Inc. ${ }^{x, 3}$ & $n$ & Inc. & $n$ & Inc. & $n$ & Inc. & $n$ & Inc. \\
\hline 1 & Aran\&Bidgol & 106 & 14.42 & 46 & 5.89 & 60 & 7.22 & 96 & 10.87 & 445 & 47.42 \\
\hline 2 & Ardestan & 215 & 114.21 & 79 & 41.26 & 20 & 10.27 & 5 & 2.52 & 39 & 19.37 \\
\hline 3 & Anarak & 0 & .00 & 0 & .00 & 0 & .00 & 0 & .00 & 2 & 9.89 \\
\hline 4 & Kashan & 377 & 15.13 & 203 & 7.23 & 197 & 6.23 & 170 & 4.77 & 320 & 7.96 \\
\hline 5 & Zavareh & 21 & 26.11 & 3 & 3.67 & 11 & 13.22 & 1 & 1.18 & 3 & 3.49 \\
\hline 6 & Badrood & 122 & 89.80 & 12 & 8.48 & 4 & 2.72 & 0 & .00 & 0 & .00 \\
\hline 7 & Maymeh & 24 & 16.07 & 35 & 19.63 & 14 & 6.57 & 9 & 3.54 & 18 & 5.93 \\
\hline 8 & Shahinshahr & 24 & 1.97 & 35 & 2.41 & 13 & .75 & 9 & .43 & 19 & .77 \\
\hline 9 & Golpayegan & 5 & .71 & 6 & .83 & 9 & 1.20 & 5 & .65 & 0 & .00 \\
\hline 10 & Natanz & 58 & 33.27 & 26 & 14.33 & 37 & 19.58 & 7 & 3.56 & 147 & 71.79 \\
\hline 11 & Naein & 25 & 9.05 & 11 & 3.86 & 6 & 2.04 & 2 & .66 & 30 & 9.61 \\
\hline 12 & Borkhar & 144 & 74.46 & 230 & 105.81 & 234 & 95.77 & 122 & 44.42 & 131 & 42.44 \\
\hline 13 & Daran & 9 & 3.19 & 5 & 1.90 & 1 & .41 & 2 & .88 & 2 & .94 \\
\hline 14 & Kohpayeh & 7 & 13.51 & 6 & 11.22 & 0 & .00 & 4 & 7.02 & 4 & 6.81 \\
\hline 15 & Chadegan & 1 & .64 & 4 & 2.69 & 1 & .70 & 3 & 2.20 & 5 & 3.83 \\
\hline 16 & Isfahan & 1552 & 7.97 & 963 & 4.79 & 432 & 2.08 & 375 & 1.75 & 977 & 4.43 \\
\hline 17 & Tiran & 8 & 3.40 & 7 & 2.87 & 10 & 3.95 & 3 & 1.14 & 18 & 6.61 \\
\hline 18 & Harand & 50 & 68.02 & 48 & 63.27 & 16 & 20.44 & 26 & 32.18 & 161 & 193.09 \\
\hline 19 & Varzaneh & 195 & 163.34 & 87 & 70.62 & 258 & 202.93 & 256 & 195.11 & 780 & 576.03 \\
\hline 20 & Felavarjan & 12 & .93 & 12 & .92 & 22 & 1.68 & 21 & 1.58 & 27 & 2.01 \\
\hline 21 & Najafabad & 41 & 1.57 & 28 & .98 & 16 & .54 & 8 & .26 & 26 & .78 \\
\hline 22 & Baghbahadoran & 1 & 1.11 & 3 & 3.11 & 2 & 1.95 & 3 & 2.74 & 0 & .00 \\
\hline 23 & Lenjan & 17 & .86 & 33 & 1.56 & 18 & .80 & 19 & .79 & 23 & .90 \\
\hline 24 & Nikabad & 25 & 61.01 & 37 & 87.49 & 23 & 52.70 & 9 & 19.98 & 3 & 6.45 \\
\hline 25 & Mobarakeh & 12 & 1.15 & 9 & .82 & 5 & .44 & 7 & .58 & 10 & .79 \\
\hline 26 & Zibashahr & 1 & 1.08 & 0 & .00 & 0 & .00 & 0 & .00 & 0 & .00 \\
\hline 27 & Hasanabad & 25 & 59.45 & 56 & 129.06 & 18 & 40.20 & 3 & 6.49 & 83 & 174.04 \\
\hline 28 & Dehaghan & 8 & 3.35 & 4 & 1.69 & 6 & 2.57 & 4 & 1.73 & 3 & 1.31 \\
\hline
\end{tabular}




\begin{tabular}{|llllllllllll|}
\hline & & $\mathbf{2 0 1 4}$ & \multicolumn{2}{c}{$\mathbf{2 0 1 5}$} & \multicolumn{2}{c}{$\mathbf{2 0 1 6}$} & \multicolumn{2}{c|}{$\mathbf{2 0 1 7}$} & \multicolumn{2}{c|}{$\mathbf{2 0 1 8}$} \\
\cline { 3 - 14 } & & $\mathbf{n}^{\mathbf{y}, 2}$ & Inc. & $\mathbf{n}$ & Inc. & $\mathbf{n}$ & Inc. & $\mathbf{n}$ & Inc. & $\mathbf{n}$ & Inc. \\
\hline 29 & Shahreza & 45 & 3.61 & 30 & 2.25 & 26 & 1.83 & 20 & 1.32 & 19 & 1.17 \\
\hline 30 & Semirom & 6 & 2.32 & 3 & 1.02 & 2 & .60 & 6 & 1.57 & 0 & .00 \\
\hline
\end{tabular}

The highest incidence of the disease occurred in the age group of 18-64 years and and the lowest occurred in the age group of 65 years and older. About $61.6 \%$ of patients were male.

According to seasonal distribution of $\mathrm{CL}$, autumn (58.56\%) and summer (26.96\%) had the highest frequency and spring $(4.05 \%)$ and winter $(10.41 \%)$ had the lowest frequency respectively during years of the study. The disease had a peak in autumn.

Figure1, which have shown the distribution map of the incidence of cutaneous leishmaniasis in Isfahan cities from 2014 to 2018 indicate that the number of cities with the frequency of leishmaniasis has decreased during these five years, so that in year of 2014, 9 cities in the northern, northeastern and southeastern parts of Isfahan province including Varzaneh, Ardestan, Badrud, Borkhar, Harand, Nik Abad, Hassan Abad, Natanz and Zavareh were involved with leishmaniasis. But in 2018, it was reduced to only four cities including Varzaneh, Natanz, Harand and Hassan Abad.

Time-trend of incidence of CL had not been stable from 2014 to 2018. Until 2017, it had both decreasing and increasing, but it has shown a declining trend in 2017 and there was a sudden upward trend of disease in 2018. The time trend has shown that Out of 9 endemic cities, only Varzaneh had always an increasing trend in these five years (except 2015) compared to other cities and has been considered as a hyperandemic region of Isfahan province [Chart1].

Figure 2 shows the analysis of hotspots to identify hot and cold spots in 2014-2018. In 2017 and 2018, Natanz and Varzaneh were considered as hot spots and Chadegan as cold spot. Varzaneh was the hottest spot (at the level of $99 \%$ ) and had the most unsuitable position in during five years and among all cities.

Table 2 shows the Moran index for each city at the optimal interval confidence, which has its highest value at the zscore peaks and the p-value was close to zero. In this study, the differences for all points were not significant ( $p$ value $>0.05$ ), and therefore non-endemic cities were not affected by endemic areas.

Table 2

Moran's Index for $\mathrm{CL}$ in the cities of Isfahan during 2014-2018.

\begin{tabular}{|c|c|c|c|c|}
\hline 2014 & 0.12 & 0.591 & 0.19 & 0.84 \\
\hline 2015 & 0.09 & 0.43 & 0.17 & 0.85 \\
\hline 2016 & 0.06 & 0.50 & 0.12 & 0.90 \\
\hline 2017 & 0.05 & 0.71 & 0.09 & 0.92 \\
\hline 2018 & 0.08 & 0.54 & 0.15 & 0.87 \\
\hline
\end{tabular}




\section{Discussion}

Leishmaniasis is one of the zoonotic diseases and it is one of the most important epidemiological diseases in Isfahan that requires effective control measures. This study is a survey of the spatial distribution of CL and the effect of environmental factors on the incidence of this disease during 5 years (2014-2018) in Isfahan province. Studies conducted using GIS can provide a clear picture of the spatial prevalence of diseases for health policy makers (Dehghani, et al 2019).

The trend of CL during 5 years was both decreasing and increasing in all cities, but in 2018, it suddenly had an upward trend and a very high increase in some cities, especially in Varzaneh (placed in southeast of Isfahan). This issue (increasing decreasing trend) is consistent with other studies in Iran. In a study by Hatami et al. that was conducted in the cities covered by Shiraz University of Medical Sciences in 2018, it was shown that the ten-year trend of leishmaniasis has had both a downward and an upward trend (Hatami, et al 2018). It seems that this trend is generally sinusoidal, and the activities of control the reservoirs, such as rodent control and preventive measures such as mosquito nets, netting, use of insect repellents, etc., can cause to reduce the leishmaniasis transmission. But in 2018 we had the highest number of leishmaniasis cases (compared to previous years) and this indicates the impact of another major factor that may be highly dependent on environmental changes. For example, in Varzaneh, the situation of leishmaniasis is dependent on the situation of Gavkhoni swamp, which requires improving the water and environmental conditions of this city more than before.

The age group of 18-64 years was the most vulnerable age group of leishmaniasis in this study. In other studies, the highest incidence of leishmaniasis was reported in the age group of children (under 10 years) (Nawaz, et al 2010; Rahim, et al 2011). However, another study conducted by Hamzavi et al., showed the most leishmaniasis cases between the ages of 20 and 29 (Hamzavi, et al 2009).

According to these studies and the results of our study, it can be concluded that although more bites occur in children, but in Isfahan province, most cases occur in young to middle-aged people. This may be due to the localization of transmission agents in different areas, which has made this age group, who spend most of their time outside the home, more vulnerable. However, the preventive measures should continue to be considered for children and adolescents up to middle age.

Men also had the most cases, which here is the same as the previous reason because of spending most of their time outdoors.

In our study, the seasonal distribution showed that the peak of the disease was in autumn and the lowest frequency was in spring. The results were consistent with the main results of other studies. It also consistent with the leishmaniasis incubation period because the most mosquito bites occur every year from early September to early November (Hatami, et al 2018; Khajehdalluei, et al 2014; Nejati, et al 2014).

Distribution of disease in 5 years of our study showed that the number of cities involved with leishmaniasis has been decreased (from 9 cities to 4 cities). Therefore, it can be said that preventive and control measures of leishmaniasis and also raising awareness, attitude and improving people's performance has been effective and has caused the cleansing of some cities. Despite of this decrease, unfortunately, some cities even had the highest record in the incidence of leishmaniasis, especially in 2018, such as Varzaneh, which was also considered as a 
hyperandemic region. The high prevalence of leishmaniasis in Varzaneh can be dependent on the environmental and natural conditions of this region and the planting of arches as the main culprit. In a study in 2008, Ready showed that climate change and environmental variables will affect the distribution of leishmaniasis by the effect of temperature on parasite development and vector competence, by the effect on the range and abundance of the sandfly species that act as vectors and affect the amount of human contact with the transmission cycles (Ready 2008).

In hotspot analysis, we concluded that hot spots were involved the central areas of the Isfahan province slightly inclined to the north and southeast of the province, which is mostly due to the presence of barren lands, swamps and constructions that provide a suitable place for mosquitoes and rodents. In 2018, Varzaneh was the hottest spot and had the worst position for leishmaniasis compared to all years and all cities. Despite the positive correlation, but the Moran's I index showed negative values means the leishmaniasis foci were surrounded by nonsimilar regions. Therefore, the high incidence points had no effect on non-endemic points, which may be due to reduced migration within the province even to neighboring cities.

\section{Declarations}

\section{Ethics Statement}

This study obtained ethical permission from the Ethical Committee, Research Deputy, IsfahanUniversity of Medical Sciences (Approval Number: 292106).

\section{Conflict of Interest}

The authors have no conflicts of interest to declare for this study.

\section{Author Contributions}

Conceptualization: MRM, FJ. Data curation: MRM, FS, AE, AH, HSK, JR, RF. Formal analysis: MRM, AE, MM. Writing - original draft: MRM, FJ, FS, AE, AH, HSK, JR, RF, MM. Writing - review \& editing: MRM, FJ, FS, AE, AH, HSK, JR, RF, MM.

Consent to Participate: All authors have consent of participate in research

Consent to Publish: All authors have consent of publish the research

Funding: the source of funding of this research was Isfahan Univesity of Medical Sciences, Isfahan, Iran.

Availability of data and materials: Data and materials can be made available upon request.

\section{References}

Abedi-Astaneh F, Hajjaran H, Yaghoobi-Ershadi MR, et al (2016) Risk Mapping and Situational Analysis of Cutaneous Leishmaniasis in an Endemic Area of Central Iran: A GIS-Based Survey. Plos one 11:e0161317.

Alvar J, Vélez ID, Bern C, Herrero M, Desjeux P, Cano J, et al (2012) Leishmaniasis worldwide and global estimates of its incidence. PloS one 7:e35671. 
Anker M, Schaaf D, Arthur R, Barkway J, Birmingham M, Burton A, et al (2000) Organization WH. WHO report on global surveillance of epidemic-prone infectious diseases. World Health Organization, 121 pp.

Artun $O$ (2019) Ecological niche modeling for the prediction of cutaneous leishmaniasis epidemiology in current and projected future in Adana, Turkey. Journal of vector borne diseases 56:127-133.

Blachowski J (2016) Application of GIS spatial regression methods in assessment of land subsidence in complicated mining conditions: case study of the Walbrzych coal mine (SW Poland). Natural Hazards 84:997-1014.

Cardoso DT, de Souza DC, de Castro VN, Geiger SM, Barbosa DS (2019) Identification of priority areas for surveillance of cutaneous leishmaniasis using spatial analysis approaches in Southeastern Brazil. BMC infectious diseases 19:318.

Cloots K, Uranw S, Ostyn B, Bhattarai NR, Le Rutte E, Khanal B, et al (2020) Impact of the visceral leishmaniasis elimination initiative on Leishmania donovani transmission in Nepal: a 10-year repeat survey. The Lancet Global Health 8: e237-e243.

Dehghani A, Lotfi MH, Falahzadeh H, VahdatK, Shabani Z (2019) Epidemiological Study and Spatial Modeling of Cutaneous Leishmaniasis in Bushehr Province using the Geographic Information System (GIS) during 2011-2015. Journal of Community Health Research 8:156-163.

Glans H, Dotevall L, Söbirk SK, Färnert A, Bradley M (2018) Cutaneous, mucocutaneous and visceral leishmaniasis in Sweden from 1996-2016: a retrospective study of clinical characteristics, treatments and outcomes. BMC infectious diseases 18:632.

Golpayegani AA, Moslem AR, Akhavan AA, Zeydabadi A, Mahvi AH, Allah-Abadi AJJoa-bd (2018) Modeling of environmental factors affecting the prevalence of zoonotic and anthroponotic cutaneous, and zoonotic visceral leishmaniasis in foci of Iran: a remote sensing and GIS based study 12: 41-66.

Hamzavi Y, Sobhi SA, Rezaei M (2009) Epidemiological factors of cutaneous lieshmaniasis in patient's reffered to health centers in Kermanshah province 2001-2006. Journal of Kermanshah University of Medical Sciences (J Kermanshah Univ Med Sci) 13: 151-161.

Hatami I, Khanjani N, Aliakbarpoor M, Dehghan A (2018) Epidemiologic Characteristics and Time Trend of Cutaneous Leishmaniasis Incidence in Cities under the Surveillance of Shiraz University of Medical Sciences. Scientific Journal of School of Public Health and Institute of Public Health Research. Spring 16:1-18.

Kavousi A, Moradi A, Rahmani K, Zeini S, Ameri P (2020) Geographical distribution of at fault drivers involved in fatal traffic collisions in Tehran, Iran. Epidemiol Health 42:e2020002.

Khajehdalluei M, Yazdanpanah MJ, Seyednozadi S, Fata A, Juya MR, Masoudi MH, Najafi M (2014) Epidemiology of cutaneous leishmaniasis in population covered by Mashhad University of medical sciences in 2011. Medical journal of Mashhad University of medical sciences 57:647-654.

Mackey TK, Liang BA, Cuomo R, Hafen R, Brouwer KC, Lee DE (2014) Emerging and reemerging neglected tropical diseases: a review of key characteristics, risk factors, and the policy and innovation environment. Clinical microbiology reviews 27:949-79. 
Mandal R, Kesari S, Kumar V, Das P ( 2018) Trends in spatio-temporal dynamics of visceral leishmaniasis cases in a highly-endemic focus of Bihar, India: an investigation based on GIS tools. Parasites \& vectors 11:220.

Masmoudi A, Hariz W, Marrekchi S, Amouri M, Turki H (2013) Old World cutaneous leishmaniasis: diagnosis and treatment. Journal of dermatological case reports 7:31-41.

Nawaz R, Khan A.M, Khan SU, Rauf A, 2010. Frequency of cutaneous leishmaniasis in an Afghan refugee camp at Peshawar. Gomal Journal of Medical Sciences 8: 16-19.

Nejati J, Mojadam M, HanafiBojd AA, Keyhani A, HabibiNodeh F (2014) An epidemiological study of Cutaneous Leishmaniasis in Andimeshk 2005-2010.Journal of llam University of medical sciences 21:94-101.

Ostad M, Shirian S, Pishro F, Abbasi T, Ai A, Azimi FJPO (2016) Control of cutaneous leishmaniasis using geographic information systems from 2010 to 2014 in Khuzestan Province, Iran 11:e0159546.

Rahim F, Jamal S, Raziq F, Uzair M, Sarvar B, Ali H, Sherin M (2011) An outbreak of Cutaneous Leishmaniasis in a village of district Dir, NWFP. Journal of Postgraduate Medical Institute (Peshawar-Pakistan) 17: 85-89 .

Ramezankhani R , Hosseini A, Sajadi N, Khoshabi M, Ramezankhani A (2017)Environmental risk factors for the incidence of Cutaneous Leishmaniasis in an endemic area of Iran: a GIS-based approach. Spatial and Spatiotemporal Epidemiology 21: 57-66.

Ready PD (2008) Leishmaniasis emergence and climate change. Scientific and Technical Review of the Office International des Epizootie 27: 399-412.

Shirzadi MR, Javanbakht M, Jesri N, Saghafipour A (2019) Spatial Distribution of Cutaneous Leishmaniasis Cases Referred to Health Centers of Three Khorasan Provinces in Iran Using Geographical Information System. Iranian Journal of Public Health 48: 1885-1892.

\section{Figures}




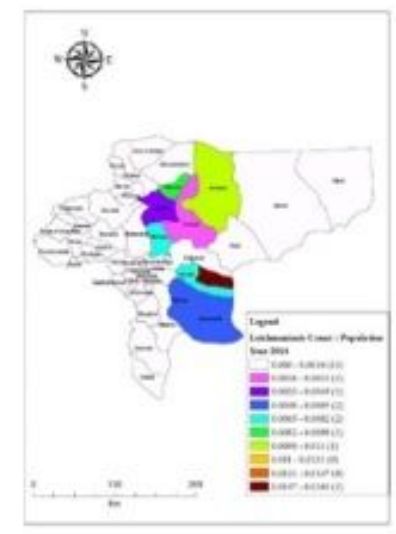

2014

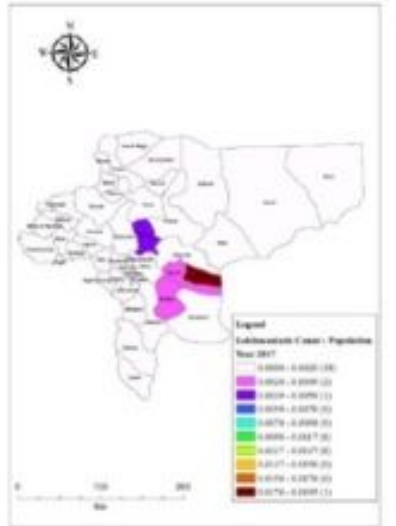

2017

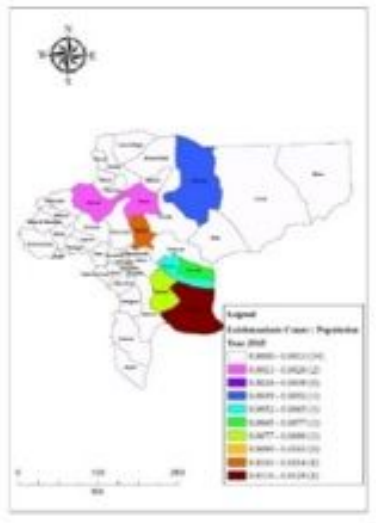

2015

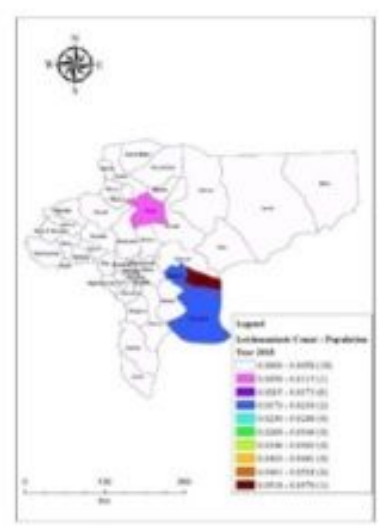

2018

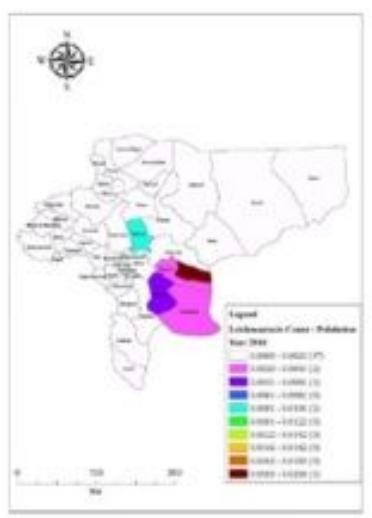

2016

\section{Figure 1}

Distribution map of the incidence of CL in the Isfahan cities respectively in 2014-2018. 


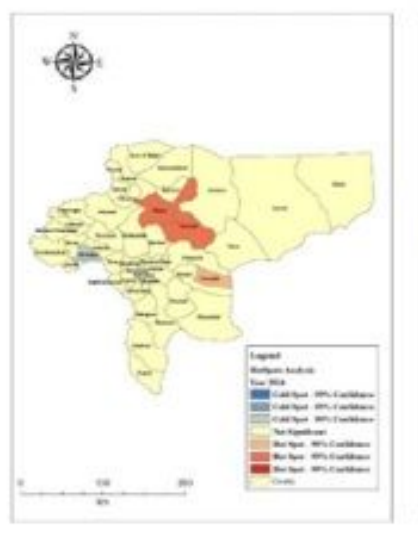

2014

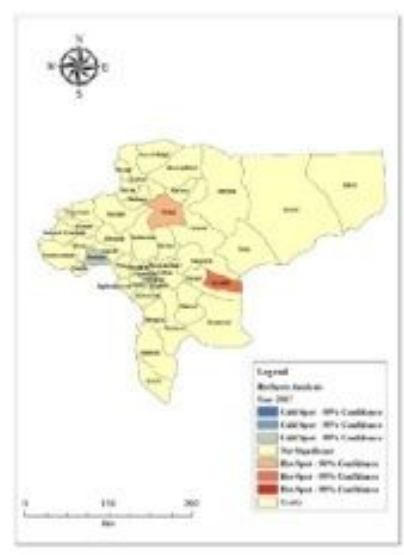

2017

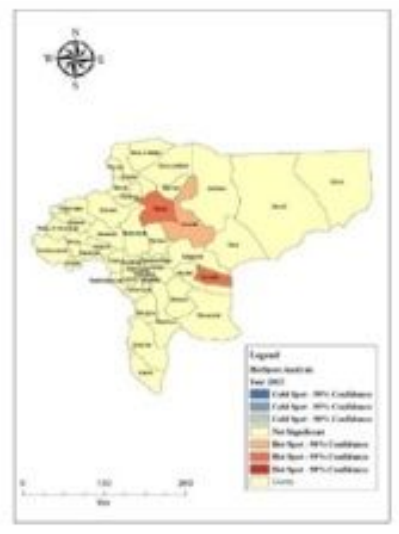

2015

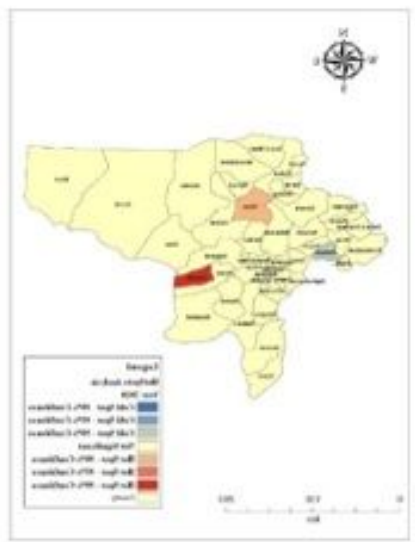

2018

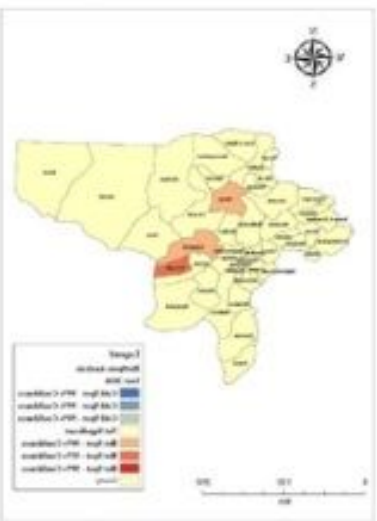

2016

\section{Figure 2}

Hot-spot analysis of CL using Getis-ord statistics in the cities of Isfahan respectively in 2014-2018.

\section{Supplementary Files}

This is a list of supplementary files associated with this preprint. Click to download.

- Chart1.pdf 